\title{
In vitro Comparative Efficacy of Various Concentrations of Aqueous and Methanolic Leaf Extract of Ocimum sanctum against Tetranychus urticae Koch in Tomato
}

\author{
Sonika Khatak*, Rachna Gulati, Monika Jangra and Anju
}

Department of Zoology and Aquaculture, Chaudhary Charan Singh Haryana Agricultural University, Hisar, Haryana 125004, India

*Corresponding author

\begin{tabular}{l} 
K e y w o r d s \\
Leaf extract, Mites, \\
Ocimum sanctum, \\
Tetranychus urticae \\
Article Info \\
Accepted: \\
$\begin{array}{l}\text { 18 April } 2020 \\
\text { Available Online: } \\
\text { 10 May } 2020\end{array}$ \\
\hline
\end{tabular}

A B S T R A C T

\begin{abstract}
In present investigation, the bioefficacy of aqueous and methanolic leaf extracts of Ocimum sanctum were tested to evaluate their toxic effects at different concentration $(10.00,7.50,5.00,2.50,2.00,1.50,1.00$ and 0.50 percent) against mixed population of Tetranychus urticae on tomato during 2018 under in vitro conditions. T. urticae responded to the mite in a concentration dependent manner i.e. lowest number of live mites and highest mortality in population was obtained with highest concentration tested (10.0\%). Out of an initial number 10 mite, significantly less number of mites were recorded at 10.00 percent concentration of $O$. sanctum leaf extract followed by $7.50,5.00,2.50$ and 2.50 per cent concentration than 1.50, 1.00 and 0.50 percent treatment and control. Direct spray bioassay results clearly revealed that aqueous and methanolic leaf extract of $O$. sanctum possessed acaricidal activity. Mites responded to extract in a concentration dependent manner. The methanolic leaf extract was more potent to T. urticae causing 20 to 71.9 percent mortality at 0.5 to 10 percent concentration as compared to aqueous leaf extract (6.7 to $61 \%$ mortality). The $\mathrm{LC}_{50}$ value in methanol extract was 3.46 percent while it was 7.06 percent in aqueous extract against $T$. urticae.
\end{abstract}

\section{Introduction}

Tomato offer food, shelter and reproductive sites (Mehta, 2012) to more than 200 insects and mites worldwide (Lange and Bronson, 1981). Two spotted spider mite, T. urticae (Acari: Tetranychidae) is an important agricultural pest of solanaceous crops including tomato in greenhouses and open fields worldwide (Awad et al., 2018). Apart from tomato, it is known to attack about 1200 species of plants, out of which more than 150 are economically important such as corn, cotton (Aucejo et al., 2003), okra (Geroh, 2011), cucumber (Kanika et al., 2013), eggplant (Sonika et al., 2017), peppers and beans (Uddin et al., 2015). It causes 70 percent damage depending on period of 
infestation on several commercial crops including peanuts, soybean, and others (Boubou et al., 2011). Under heavy infestations of $T$. urticae, tomato plants suffer massive leaf drop and yield loss. Because of the negative impact of $T$. urticae on tomato crops, various management practices are followed against mites. There are several reports on botanicals like, neem (MartinezVillar et al., 2005), common tancy, wormwood extracts (Chiasson et al., 2001), aak, oleander (Islam et al., 2008), marigold, garlic, pepper (Boyd and Alverson, 2000; Rusch, et al., 2010), which are effective against $T$. urticae adults.

Tulsi (Holy Basil), Ocimum genus is a traditional plant considered sacred by the Hindus. It has small stems and leaves with an appreciable aroma due to essential oils, such as eugenol, methyl-chavicol, methyl-eugenol, saffron, geranial, thymol and linalool (Deshpande et al., 1997; Blank et al., 2005). Its use as an insecticide, nematicide, fungicide and antimicrobial compound has been reported (Mishra and Mishra, 2011). Leaf extracts of $O$. sanctum caused 100 and 75 per cent mortality in mushroom mite, Luciaphorus sp. (Bussaman et al., 2012) and T. urticae (Kanniammal and Chinniah, 2012), respectively. It showed 65 per cent repellency against Petrobia harti, another tetranychid mite (Mitra et al., 2015).

Water extract of O. basicilum (@15\%) caused 74 percent adult mortality and complete inhibition of $T$. urticae fecundity (Isabel et al., 2016). Ogayo et al., (2019) reported that plant extract of $O$. gratissimum and Leonotise nepetifolia at 12 percent concentration showed highest efficacy (82.75 and $69.06 \%$ ) against $T$. urticae which led to increase in pod numbers and pod yield in French bean. Keeping these views in mind, the present study was made to study the effect of aqueous and methanolic extracts of leaves of tulsi
(Ocimum sactum) for management of this pest of tomato.

\section{Materials and Methods}

Under in vitro conditions, bio-efficacy of aqueous and methanolic leaf extracts of Ocimum sanctum were evaluated against $T$. urticae to determine their acaricidal activity against $T$. urticae under standardized conditions $(30 \pm 10 \mathrm{C}, 80-85 \% \mathrm{RH})$ in the Acarology Lab, Department of Zoology, CCS HAU during, 2018. Infested leaves were plucked from the field crop and brought to the laboratory. Under stereo zoom microscope, mobile stages of mite were picked with the help of bird's feather pick and released on the separate untreated leaf. Each subset was replicated three times containing 10 mites in each replicate.

\section{Efficacy of Ocimum sanctum against Tetranychus urticae}

Aqueous and Methanolic Leaf extract Tulsi (Ocimum sanctum) were evaluated for bioeffficacy studies against $T$. urticae using Leaf Disc Technique. In this technique, a moist cotton bed was prepared in a petri plate with covering lid. The bed was circled with moist cotton pad to prevent the escape of mites. This leaf was kept above the moist cotton pad in the centre of the petri plate. The extracts were sprayed directly on tomato leaves after releasing the mites on them and daily number of mites was counted.

Preparation of aqueous and methanolic leaf extract of Ocimum sanctum

Coarsely ground leaves $(250 \mathrm{~g})$ of $O$. sanctum were soaked in 250 litre of distilled water for 48 hours at room temperature. Intermittent shaking was done during this period. Later, the solution was filtered through the muslin cloth and stored in glass bottle for use in 
experimentation. This stock was considered as 100 percent and further dilutions were made by adding distilled water to obtain 10 , $7.5,5,2.5,2,1.5,1$ and 0.5 percent concentrations.

Methanolic extract of leaves were prepared following the standard procedure of refluxing and distillation (Kumar et al., 2001). Leaves were cleaned and were allowed to shade-dry for a month, after which they were crushed into bits. Crushed leaves (250 g) were taken in a round bottom flask of 2 litre capacity and methanol AR grade was added to it to immerse the bits and kept it for overnight. Refluxing was done by fitting the flask with a water condenser and boiling the set using a heating mantle for $8 \mathrm{~h}$. The extract was then filtered out of the flask and concentrated by distillation process. This procedure of refluxing and distillation was repeated thrice for complete extraction of active ingredients from leaves. In this process 300 to $250 \mathrm{ml}$ of methanolic extracts were obtained respectively. This stock was considered as 100 percent and further dilutions were made by adding methanol to obtain different concentrations viz., 10.00, 7.50, 5.00, 2.50, $2.00,1.50,1.00$ and 0.50 percent of methanolic extracts. Suitable control without water and with water was maintained for the experiments.

\section{Direct spray technique}

The leaves from untreated and uninfested healthy plants were used in these experiments. Under stereo zoom microscope, mobile stages of mites were picked with the help of bird's feather pick and released on the separate untreated leaf disc. Before releasing the mites, leaves were washed properly. Separate sets were maintained for aqueous and methanolic leaf extract. Each set contained 8 subsets of different concentrations (treatment) and two controls. Similarly, subsets of different concentrations were prepared for methanolic extracts. Each subset was replicated three times containing 10 mites per replicate. Each concentration was sprayed separately on leaf with the help of hand automizer. The leaf was placed on moist cotton bed of the covered petri plate.

Observations on the live mite stages were recorded after every $24 \mathrm{~h}$ under microscope in each treatment. These were compared with control. Before considering the mite stage as dead, it was probed lightly with the help of bird's feather pick to detect any movement. Observations were continued after every $24 \mathrm{~h}$ till the appearance of the next stage or mortality of test stage. The observed mortality was converted into percent mortality (\% reduction) and corrected mortality was obtained after deducting the mortality in control treatments. The data were used for calculating the $\mathrm{LC}_{50}$ of both the extracts.

The percent reduction in mite count as compared to pre- treatment count was calculated by the formula:

Percent Reduction $=$

(Pre-treatment count - Number of live mites after treatment)

Pre- treatment count

\section{Results and Discussion}

\section{Statistical analysis}

For assessing the effectiveness of the treatments, mean numbers of $T$. urticae were pooled and analyzed statistically. Critical difference (CD) was calculated between the treatments to see the impact of population buildup of $T$. urticae on tomato by single and factorial CRD (in vitro) method. Data for evaluating the effect of $O$. sanctum against $T$. 
urticae under in vitro conditions was subjected to two factorial CRD. Data transformation was applied wherever necessary. CD was calculated in each case and means of treatments were compared to see the significant difference between the treatments and with control at different observation periods. CD was also used to find out the most effective extract and its concentration.

Bioefficacy of aqueous and methanolic extract of Ocimum sanctum leaves were evaluated at different concentrations against mixed population of $T$. urticae in tomato during 2018 under in vitro and in vivo conditions. The results revealed that all the treatments possessed acaricidal activity against $T$. urticae but to different extents. The obtained results have been summarized in Tables and figures are described below:

\section{Aqueous leaf extracts of Ocimum sanctum}

Direct spray bioassay results clearly revealed that aqueous leaf extract of $O$. sanctum possessed acaricidal activity. Mites responded to extract in a concentration dependent manner i.e. lowest number $(4.29$ mites $/ \mathrm{sq} \mathrm{cm}$ leaflet) of live mites was obtained with highest concentration (10\%) and highest number (9.33 mites/sq cm leaflet) with lowest concentration $(0.5 \%)$ (Table 1). All the treatments were significantly better than controls (9.67, $9.43 \mathrm{mites} / \mathrm{sq} \mathrm{cm}$ leaflet) $(\mathrm{CD}=0.08 ; \mathrm{p}=0.05)$. Both the control treatments, water sprayed and unsprayed leaves along with the lower two $O$. sanctum aqueous extract treatments $(9.29,9.33$ mites/sq cm leaflet) were statistically comparable with each other. This suggested that water treatment did not cause significant mortality in mites. The treatments, 5.0 (6.57 mites/sq cm leaflet) and 2.5 (6.86 mites/sq cm leaflet) per cent, 2.0 (7.52 mites/sq cm leaflet) and 1.5 (8.00 mites/sq cm leaflet) per cent were statistically comparable in terms of mean number of mites. Statistical analysis revealed a significant effect of observation period. The number of mites significantly decreased to 9.40 after first day of spray as compared to initial 10 mites $(\mathrm{CD}=0.07$; $\mathrm{p}=0.05)$. After $2^{\text {nd }}$ and $3^{\text {rd }}$ day of spray, the number of mites decreased to 9.07 and 8.67 mites; showing non significant decrease with each other.

Thereafter, significantly lower numbers of mites (8.10, 7.43, 6.07 and 4.30) were recorded after 4, 5, 6 and 7 day of spray. The ANOVA revealed significant interaction between the treatment and observation periods $(\mathrm{CD}=0.22 ; \mathrm{p}=0.05)$ (Table 1$)$. This showed that at each observation period, higher concentrations were more effective in reducing the $T$. urticae population than lower concentrations.

\section{Methanolic leaf extracts of Ocimum sanctum}

Similar results were obtained with methanolic leaf extract of $O$. sanctum showing significantly lower number of mites as compared with control $(9.29,9.81 \mathrm{mites} / \mathrm{sq}$ $\mathrm{cm}$ leaflet in water spray and unsprayed leaf disc, respectively) $(\mathrm{CD}=0.09 ; \mathrm{p}=0.05)$. Both the control treatment did not cause any significant mortality in T. urticae and were at par with each other. Among the treatments, higher reductions $(2.81,3.57 \mathrm{mites} / \mathrm{sq} \mathrm{cm}$ leaflet) were witnessed at higher concentrations $(10,7.5 \%)$.

The number of mites was 5.14, 5.48, 6.00, 6.76, 6.91 and $8.00 \mathrm{mites} / \mathrm{sq} \mathrm{cm}$ leaflet at 5.0, $2.5,2.0,1.5,1.0$ and 0.5 percent of methanolic extract (Table 2). The concentrations, 5 and 2.5, 1.5 and 1.0 per cent were at par with each other. A significant effect of observation period was recorded in this bioassay. First day after the spray, 
number of mites significantly decreased to 8.37 as compared to initial 10 mites $(\mathrm{CD}=0.08 ; \mathrm{p}=0.05)$. Thereafter it showed a significant gradual decrease to $7.93,7.43$, $6.47,5.73,4.87$ and 3.83 after $2^{\text {nd }}, 3^{\text {rd }}, 4^{\text {th }}, 5^{\text {th }}$, $6^{\text {th }}$ and $7^{\text {th }}$ day spray. The number of mites recorded after $1^{\text {st }}$ and $2^{\text {nd }}$ day was statistically comparable. Likewise, the numbers of mites observed after $2^{\text {nd }}$ and $3^{\text {rd }}$ day of spray do not differ significantly with each other. Interaction between the treatment and observation periods was found significant $(\mathrm{CD}=0.25 ; \mathrm{p}=0.05$ ) (Table 2). This showed that at the end of study period ( $7^{\text {th }}$ day), lower T. urticae number was observed at higher concentrations than at lower concentration.

Table.1 Estimation of vitamin B-6 (ppm) in Areca nut collected from different locations of Karnataka

\begin{tabular}{|c|c|c|c|c|c|c|c|c|c|}
\hline \multirow{2}{*}{$\begin{array}{l}\text { Concentration } \\
(\%)\end{array}$} & \multirow{2}{*}{$\begin{array}{c}\text { Pre } \\
\text { treatment } \\
\text { count }\end{array}$} & \multicolumn{7}{|c|}{ Number of live mites/leaf disc } & \multirow[t]{2}{*}{ Mean } \\
\hline & & $\mathbf{1}^{\mathrm{st}}$ & $\begin{array}{l}2^{\text {nd }} \\
\text { day }\end{array}$ & $\begin{array}{l}3^{\text {rd }} \\
\text { day }\end{array}$ & $\begin{array}{l}4^{\text {th }} \\
\text { day }\end{array}$ & $\begin{array}{l}5^{\text {th }} \\
\text { day }\end{array}$ & $\begin{array}{c}6^{\text {th }} \\
\text { day }\end{array}$ & $\begin{array}{l}7^{\text {th }} \\
\text { day }\end{array}$ & \\
\hline 10.0 & 10.00 & $\begin{array}{c}7.67 \\
(2.94)\end{array}$ & $\begin{array}{c}7.00 \\
(2.82)\end{array}$ & $\begin{array}{c}6.33 \\
(2.70)\end{array}$ & $\begin{array}{c}4.33 \\
(2.31) \\
\end{array}$ & $\begin{array}{c}3.00 \\
(1.99)\end{array}$ & $\begin{array}{c}1.33 \\
(1.49)\end{array}$ & $\begin{array}{c}0.33 \\
(1.14)\end{array}$ & $\begin{array}{c}4.29 \\
(2.20) \\
\end{array}$ \\
\hline 7.5 & 10.00 & $\begin{array}{c}8.33 \\
(3.05)\end{array}$ & $\begin{array}{c}7.67 \\
(2.94)\end{array}$ & $\begin{array}{c}6.33 \\
(2.71)\end{array}$ & $\begin{array}{c}5.33 \\
(2.52)\end{array}$ & $\begin{array}{c}4.33 \\
(2.31)\end{array}$ & $\begin{array}{c}1.67 \\
(1.63)\end{array}$ & $\begin{array}{c}0.00 \\
(1.00)\end{array}$ & $\begin{array}{c}4.81 \\
(2.31)\end{array}$ \\
\hline 5.0 & 10.00 & $\begin{array}{c}9.00 \\
(3.16)\end{array}$ & $\begin{array}{c}8.67 \\
(3.11)\end{array}$ & $\begin{array}{c}8.33 \\
(3.05)\end{array}$ & $\begin{array}{c}7.67 \\
(2.94)\end{array}$ & $\begin{array}{c}7.00 \\
(2.83)\end{array}$ & $\begin{array}{c}4.67 \\
(2.38)\end{array}$ & $\begin{array}{c}0.67 \\
(1.24)\end{array}$ & $\begin{array}{c}6.57 \\
(2.67)^{\mathrm{a}}\end{array}$ \\
\hline 2.5 & 10.00 & $\begin{array}{c}9.33 \\
(3.21)\end{array}$ & $\begin{array}{c}9.33 \\
(3.21)\end{array}$ & $\begin{array}{c}9.00 \\
(3.16)\end{array}$ & $\begin{array}{c}7.67 \\
(2.94)\end{array}$ & $\begin{array}{c}6.67 \\
(2.76)\end{array}$ & $\begin{array}{c}4.33 \\
(2.31)\end{array}$ & $\begin{array}{c}1.67 \\
(1.63)\end{array}$ & $\begin{array}{c}6.86 \\
(2.75)^{\mathrm{a}}\end{array}$ \\
\hline 2.0 & 10.00 & $\begin{array}{r}10.00 \\
(3.32)\end{array}$ & $\begin{array}{c}9.33 \\
(3.21)\end{array}$ & $\begin{array}{c}8.67 \\
(3.11)\end{array}$ & $\begin{array}{c}8.33 \\
(3.05)\end{array}$ & $\begin{array}{c}7.67 \\
(2.94)\end{array}$ & $\begin{array}{c}6.33 \\
(2.70)\end{array}$ & $\begin{array}{c}2.33 \\
(1.82)\end{array}$ & $\begin{array}{c}7.52 \\
(2.88)^{b}\end{array}$ \\
\hline 1.5 & 10.00 & $\begin{array}{r}10.00 \\
(3.32)\end{array}$ & $\begin{array}{c}9.67 \\
(3.27)\end{array}$ & $\begin{array}{c}9.00 \\
(3.16)\end{array}$ & $\begin{array}{c}8.67 \\
(3.11)\end{array}$ & $\begin{array}{c}8.00 \\
(3.00)\end{array}$ & $\begin{array}{c}6.33 \\
(2.71)\end{array}$ & $\begin{array}{c}4.33 \\
(2.31)\end{array}$ & $\begin{array}{c}8.00 \\
(2.98)^{b}\end{array}$ \\
\hline 1.0 & 10.00 & $\begin{array}{c}9.67 \\
(3.27)\end{array}$ & $\begin{array}{c}9.67 \\
(3.27)\end{array}$ & $\begin{array}{c}9.67 \\
(3.27)\end{array}$ & $\begin{array}{c}9.67 \\
(3.27)\end{array}$ & $\begin{array}{c}9.33 \\
(3.21)\end{array}$ & $\begin{array}{c}9.00 \\
(3.16)\end{array}$ & $\begin{array}{c}8.00 \\
(3.00)\end{array}$ & $\begin{array}{c}9.29 \\
(3.21)^{c}\end{array}$ \\
\hline 0.5 & 10.00 & $\begin{array}{l}10.00 \\
(3.32)\end{array}$ & $\begin{array}{c}9.67 \\
(3.27)\end{array}$ & $\begin{array}{c}9.67 \\
(3.27)\end{array}$ & $\begin{array}{c}9.67 \\
(3.27)\end{array}$ & $\begin{array}{c}9.33 \\
(3.21)\end{array}$ & $\begin{array}{c}9.00 \\
(3.16)\end{array}$ & $\begin{array}{c}8.00 \\
(3.00)\end{array}$ & $\begin{array}{c}9.33 \\
(3.21)^{c}\end{array}$ \\
\hline $\begin{array}{l}\text { Control } \\
\text { (with water) }\end{array}$ & 10.00 & $\begin{array}{l}10.00 \\
(3.32)\end{array}$ & $\begin{array}{c}9.67 \\
(3.27)\end{array}$ & $\begin{array}{c}9.67 \\
(3.27)\end{array}$ & $\begin{array}{c}9.67 \\
(3.27)\end{array}$ & $\begin{array}{c}9.33 \\
(3.21)\end{array}$ & $\begin{array}{c}9.00 \\
(3.16)\end{array}$ & $\begin{array}{c}8.67 \\
(3.11)\end{array}$ & $\begin{array}{c}9.43 \\
(3.23)^{c}\end{array}$ \\
\hline $\begin{array}{l}\text { Control } \\
\text { (Unsprayed) }\end{array}$ & 10.00 & $\begin{array}{l}10.00 \\
(3.32)\end{array}$ & $\begin{array}{l}10.00 \\
(3.32)\end{array}$ & $\begin{array}{l}10.00 \\
(3.32)\end{array}$ & $\begin{array}{l}10.00 \\
(3.32)\end{array}$ & $\begin{array}{c}9.67 \\
(3.27)\end{array}$ & $\begin{array}{c}9.00 \\
(3.16)\end{array}$ & $\begin{array}{c}9.00 \\
(3.16)\end{array}$ & $\begin{array}{c}9.67 \\
(3.27)^{c}\end{array}$ \\
\hline Mean & 10.00 & $\begin{array}{c}9.40 \\
(3.22)^{b}\end{array}$ & $\begin{array}{c}9.07 \\
(3.17)^{a, b}\end{array}$ & $\begin{array}{c}8.67 \\
(3.10)^{a}\end{array}$ & $\begin{array}{c}8.10 \\
(3.00)\end{array}$ & $\begin{array}{c}7.43 \\
(2.87)\end{array}$ & $\begin{array}{c}6.07 \\
(2.59)\end{array}$ & $\begin{array}{c}4.30 \\
(2.14)\end{array}$ & \\
\hline
\end{tabular}

Figures in parentheses are $\sqrt{\mathrm{n}}+1$ transformation

C.D. for Treatment $(\mathrm{T})=(0.08), \mathrm{SE}(\mathrm{m})=(0.03)$;

C.D. for Observation Period $(\mathrm{OP})=(0.07), \mathrm{SE}(\mathrm{m})=(0.03)$

C.D. for Interaction $\mathrm{OP} \times \mathrm{T}=(0.22), \mathrm{SE}(\mathrm{m})=(0.08)$;

Values with the same superscript do not differ significantly 
Table.2 In vitro bioassay of Ocimum sanctum methanolic leaf extract against Tetranychus urticae

\begin{tabular}{|c|c|c|c|c|c|c|c|c|c|}
\hline \multirow{2}{*}{$\begin{array}{c}\text { Concentration } \\
(\%)\end{array}$} & \multirow{2}{*}{$\begin{array}{l}\text { Pretreatment } \\
\quad \text { count }\end{array}$} & \multicolumn{7}{|c|}{ Number of live mites/leaf disc } & \multirow{2}{*}{ Mean } \\
\hline & & $\begin{array}{l}1^{\text {st }} \\
\text { day }\end{array}$ & $\begin{array}{l}2^{\text {nd }} \\
\text { day }\end{array}$ & $\begin{array}{l}3^{\text {rd }} \\
\text { day }\end{array}$ & $\begin{array}{l}4^{\text {th }} \\
\text { day }\end{array}$ & $\begin{array}{c}5^{\text {th }} \\
\text { day }\end{array}$ & $\begin{array}{l}6^{\text {th }} \\
\text { day }\end{array}$ & $\begin{array}{l}7^{\text {th }} \\
\text { day }\end{array}$ & \\
\hline 10.0 & 10.00 & $\begin{array}{l}7.00 \\
(2.83)\end{array}$ & $\begin{array}{c}5.67 \\
(2.58)\end{array}$ & $\begin{array}{l}4.00 \\
(2.23)\end{array}$ & $\begin{array}{c}2.33 \\
(1.82)\end{array}$ & $\begin{array}{c}0.67 \\
(1.28)\end{array}$ & $\begin{array}{c}0.00 \\
(1.00)\end{array}$ & $\begin{array}{c}0.00 \\
(1.00)\end{array}$ & $\begin{array}{c}2.81 \\
(1.82)\end{array}$ \\
\hline 7.5 & 10.00 & $\begin{array}{l}7.67 \\
(2.94)\end{array}$ & $\begin{array}{c}6.33 \\
(2.71)\end{array}$ & $\begin{array}{c}5.67 \\
(2.58)\end{array}$ & $\begin{array}{c}3.33 \\
(2.06)\end{array}$ & $\begin{array}{c}1.67 \\
(1.63)\end{array}$ & $\begin{array}{c}0.33 \\
(1.14)\end{array}$ & $\begin{array}{c}0.00 \\
(1.00)\end{array}$ & $\begin{array}{c}3.57 \\
(2.01)\end{array}$ \\
\hline 5.0 & 10.00 & $\begin{array}{c}8.00 \\
(3.00)\end{array}$ & $\begin{array}{l}7.67 \\
(2.94)\end{array}$ & $\begin{array}{c}7.00 \\
(2.83)\end{array}$ & $\begin{array}{l}5.00 \\
(2.44)\end{array}$ & $\begin{array}{c}4.67 \\
(2.38)\end{array}$ & $\begin{array}{c}2.67 \\
(1.91)\end{array}$ & $\begin{array}{c}1.00 \\
(1.38)\end{array}$ & $\begin{array}{c}5.14 \\
(2.41)^{\mathrm{a}}\end{array}$ \\
\hline 2.5 & 10.00 & $\begin{array}{l}7.67 \\
(2.94)\end{array}$ & $\begin{array}{l}7.33 \\
(2.89)\end{array}$ & $\begin{array}{c}6.67 \\
(2.76)\end{array}$ & $\begin{array}{c}6.33 \\
(2.71)\end{array}$ & $\begin{array}{c}5.00 \\
(2.44)\end{array}$ & $\begin{array}{c}3.67 \\
(2.14)\end{array}$ & $\begin{array}{c}1.67 \\
(1.63)\end{array}$ & $\begin{array}{c}5.48 \\
(2.50)^{\mathrm{a}}\end{array}$ \\
\hline 2.0 & 10.00 & $\begin{array}{c}8.00 \\
(3.00)\end{array}$ & $\begin{array}{l}7.67 \\
(2.94)\end{array}$ & $\begin{array}{c}7.33 \\
(2.89)\end{array}$ & $\begin{array}{c}6.00 \\
(2.62)\end{array}$ & $\begin{array}{c}6.00 \\
(2.62)\end{array}$ & $\begin{array}{l}4.67 \\
(2.38)\end{array}$ & $\begin{array}{c}2.33 \\
(1.82)\end{array}$ & $\begin{array}{c}6.00 \\
(2.62)\end{array}$ \\
\hline 1.5 & 10.00 & $\begin{array}{c}8.33 \\
(3.05)\end{array}$ & $\begin{array}{c}8.33 \\
(3.05)\end{array}$ & $\begin{array}{c}7.67 \\
(2.94)\end{array}$ & $\begin{array}{c}7.33 \\
(2.89)\end{array}$ & $\begin{array}{c}6.67 \\
(2.76)\end{array}$ & $\begin{array}{c}5.67 \\
(2.58)\end{array}$ & $\begin{array}{c}3.33 \\
(2.08)\end{array}$ & $\begin{array}{c}6.76 \\
(2.77)^{b}\end{array}$ \\
\hline 1.0 & 10.00 & $\begin{array}{c}8.67 \\
(3.11)\end{array}$ & $\begin{array}{c}8.33 \\
(3.05)\end{array}$ & $\begin{array}{c}8.00 \\
(3.00)\end{array}$ & $\begin{array}{c}6.67 \\
(2.76)\end{array}$ & $\begin{array}{c}6.33 \\
(2.71)\end{array}$ & $\begin{array}{c}5.67 \\
(2.58)\end{array}$ & $\begin{array}{c}4.67 \\
(2.38)\end{array}$ & $\begin{array}{c}6.91 \\
(2.80)^{b}\end{array}$ \\
\hline 0.5 & 10.00 & $\begin{array}{c}9.00 \\
(3.16)\end{array}$ & $\begin{array}{c}8.67 \\
(3.11)\end{array}$ & $\begin{array}{c}8.67 \\
(3.11)\end{array}$ & $\begin{array}{c}8.33 \\
(3.05)\end{array}$ & $\begin{array}{c}7.33 \\
(2.89)\end{array}$ & $\begin{array}{l}7.00 \\
(2.83)\end{array}$ & $\begin{array}{c}7.00 \\
(2.83)\end{array}$ & $\begin{array}{c}8.00 \\
(3.00)\end{array}$ \\
\hline $\begin{array}{l}\text { Control(with } \\
\text { water) }\end{array}$ & 10.00 & $\begin{array}{c}9.33 \\
(3.21)\end{array}$ & $\begin{array}{c}9.33 \\
(3.21)\end{array}$ & $\begin{array}{c}9.33 \\
(3.21)\end{array}$ & $\begin{array}{c}9.33 \\
(3.21)\end{array}$ & $\begin{array}{c}9.33 \\
(3.21)\end{array}$ & $\begin{array}{c}9.33 \\
(3.21)\end{array}$ & $\begin{array}{c}9.00 \\
(3.16)\end{array}$ & $\begin{array}{c}9.29 \\
(3.21)^{\mathrm{c}}\end{array}$ \\
\hline $\begin{array}{c}\text { Control( } \\
\text { unsprayed) }\end{array}$ & 10.00 & $\begin{array}{l}10.00 \\
(3.32)\end{array}$ & $\begin{array}{l}10.00 \\
(3.32)\end{array}$ & $\begin{array}{l}10.00 \\
(3.32)\end{array}$ & $\begin{array}{l}10.00 \\
(3.32)\end{array}$ & $\begin{array}{c}9.67 \\
(3.27)\end{array}$ & $\begin{array}{c}9.67 \\
(3.27)\end{array}$ & $\begin{array}{c}9.33 \\
(3.21)\end{array}$ & $\begin{array}{c}9.81 \\
(3.29)^{c}\end{array}$ \\
\hline Mean & 10.00 & $\begin{array}{c}8.37 \\
(3.06)^{\mathrm{a}}\end{array}$ & $\begin{array}{c}7.93 \\
(2.98)^{a} \\
\text { be }\end{array}$ & $\begin{array}{c}7.43 \\
(2.89)^{\mathrm{a}}\end{array}$ & $\begin{array}{c}6.47 \\
(2.69)\end{array}$ & $\begin{array}{c}5.73 \\
(2.52)\end{array}$ & $\begin{array}{c}4.87 \\
(2.30)\end{array}$ & $\begin{array}{c}3.83 \\
(2.05)\end{array}$ & \\
\hline
\end{tabular}

Figures in parentheses are $\sqrt{n}+1$ transformation

C.D. for Treatment $(\mathrm{T})=(0.09), \mathrm{SE}(\mathrm{m})=(0.03)$;

C.D. for Observation Period $(\mathrm{OP})=(0.08), \mathrm{SE}(\mathrm{m})=(0.03)$

C.D. for Interaction $\mathrm{OP} \times \mathrm{T}=(0.25), \mathrm{SE}(\mathrm{m})=(0.08)$;

Values with the same superscript do not differ significantly

Table.3 $\mathrm{LC}_{50}$ of Ocimum sanctum extract for Tetranychus urticae

\begin{tabular}{|c|c|c|c|c|c|c|c|}
\hline \multirow{2}{*}{$\begin{array}{c}\text { Ocimum sanctum leaf } \\
\text { extract }\end{array}$} & \multicolumn{5}{|c|}{ Direct spray bioassay (Leaf disc method) } \\
\cline { 2 - 7 } & $\mathbf{n}$ & Slope & Intercept & LC $_{\mathbf{5 0}}(\boldsymbol{\%})$ & $\boldsymbol{\chi} \mathbf{2}$ & Df \\
\hline Aqueous extract & 10 & 1.25 & 3.93 & 7.06 & 12.59 & 5 \\
\hline Methanolic extract & 10 & 1.22 & 4.29 & 3.46 & 12.59 & 5 \\
\hline
\end{tabular}


Fig.1 Comparative efficacy of aqueous and methanolic leaf extract of Ocimum sanctum against Tetranychus urticae

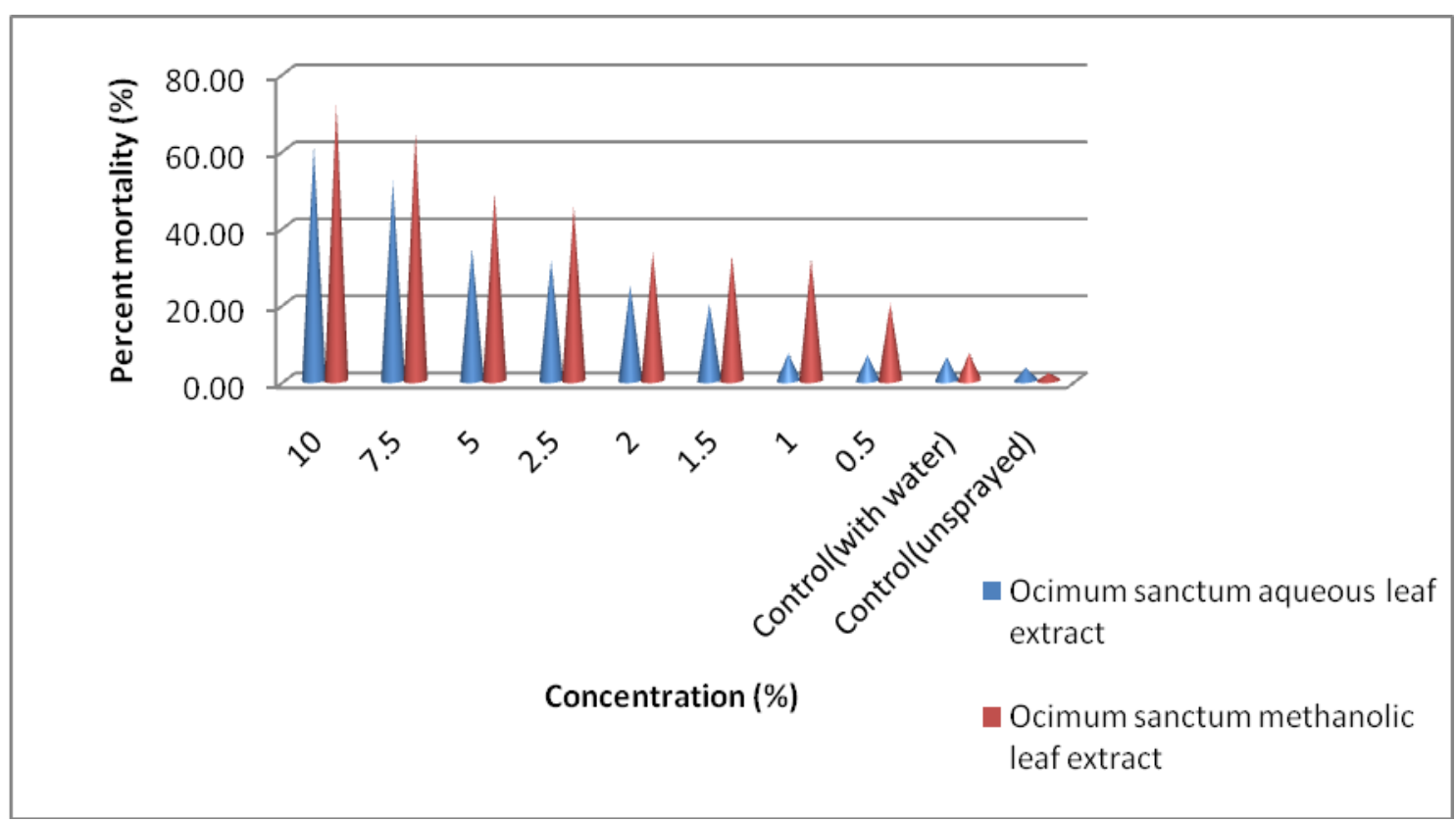

An analysis through graphical representation showed the comparative efficacy of aqueous and methanolic extracts of $O$. sanctum against T. urticae at different concentrations (Fig. 1). The higher mite mortality in methanolic leaf extract showed that it is more potent to $T$. urticae than aqueous leaf extract causing 71.9 to 20 percent mortality at 10 to 0.5 percent concentration as compared to 61 to 6.7 percent mortality in aqueous extract.

\section{Determination of concentration-mortality response $\left(\mathrm{LC}_{\mathbf{5 0}}\right)$}

The $\mathrm{LC}_{50}$ values (concentration at which 50 percent mortality occur in $T$. urticae population) along with regression statistics under direct spray bioassay were calculated using standard probit analysis method and are presented in Table 3 for both extracts of $O$. sanctum. The $\mathrm{LC}_{50}$ value in methanol extract was 3.46 percent while it was 7.06 percent in aqueous extract against $T$. urticae. The value of slope (1.25 and 1.22) was less than for both the extracts. This showed that further increase in concentrations will lead to significant decrease in number of $T$. urticae. The intercept value was 3.93 and 4.29, respectively in both the extract. The present study showed that methanolic extract was more effective against $T$. urticae than aqueous extract because of lower $\mathrm{LC}_{50}$ value.

Tulsi (Ocimum sanctum) is a traditional plant which possesses important nematicide, fungicide and insecticidal properties (Deshpande et al., 1997; Nanasombat and Lohasupthawee, 2005; Mishra and Mishra 2011). Phenolic of botanicals compounds play an important role in herbivore-host plant interaction; plants with a high concentration of phenols are often less attractive hosts for many herbivorous insects and mites than plants with a low content of these secondary metabolites (Gogoi et al., 2001; Sahayaraj et al., 2003). Secondary metabolites present in plants with insecticidal effects may act as inhibitors of insect feeding or hinder growth, development, and reproduction (Spochacz et al., 2018). 
In present study aqueous and methanolic extract of $O$. sanctum were used for control of $T$. urticae. Mites responded to $O$. sanctum aqueous and methanolic leaf extracts in a concentration dependent manner in present study i.e. lowest number (4.29 mites/sq cm leaflet) of live mites was obtained with highest concentration (10\%) and highest number (9.33 mites/sq cm leaflet) with lowest concentration $(0.5 \%)$. The present study showed that methanolic extract (3.46 percent) was more effective against $T$. urticae than aqueous extract (7.06 percent) because of lower $\mathrm{LC}_{50}$ value. Methanolic extracts Ocimum tenuiflorum (3\%) exhibited acaricidal activities against Tetranychus neocaledonicus and resulted in the 82.2 percent (Chayengia et al., 2010) and 93.3 percent mortality at (Roy et al., 2011) mortality. Kanniammal and chinniah (2012) also reported that $O$. sanctum caused highest mortality of $T$. urticae (75.07\%). Isabel et al., (2016) worked on nine Brazilian accessions. They found that, one basil accession, OVRS showed high toxicity against $T$. urticae females at 15 percent concentration waterextract; the mortality rate was 75 percent and complete inhibition of fecundity was found on peanut. Ocimum sp. also showed acaricidal effects on several parasite mites. Kamaraj et al., (2008) Ocimum gratissimum L. oil and its constituents have fumigant and repellent activity against storage pests and mites (Kim et al., 2003; Ogendo et al., 2008) as well as malaria vectors Aedes aegypti L. (Diptera: Culicidae) and Culex quinquefasciatus Say (Diptera). The essential oils of Ocimum basilicum Linn., Coriandrum sativum Linn., Eucalyptus globulus Labill, Mentha piperita Linn. and Satureja hortensis Linn. were toxic to poultry red mite [Dermanyssus gallinae (De Geer)], and, when using the in vitro direct contact method, these essential oils at the dose of $0.6 \mathrm{mg} / \mathrm{cm}$ could result in mortality rates over 80 percent after $24 \mathrm{~h}$ of contract (Magdas et al., 2010). Manzoor et al., (2011) reported that O.sanctum oil as toxicant and repellent agent against termite, Heterotermes indicola

In conclusion, the present study focused upon management practices against $T$. urticae in tomato. $10.0 \%$ concentration of both extract was most potent against T. urticae. More studies are required in this area and $O$. sanctum leaf extract can be used for protection of tomato crops against $T$. urticae.

\section{Acknowledgement}

Financial help and necessary facilities provided by Department of Zoology, CCS Haryana Agricultural University, Hisar are fully acknowledged.

\section{References}

Aucejo, S., Foo, M., Gimeno, E., Gomez, A., Montort, R., Predes, E., Ramis, M., Ripolles, J.L., and Tirado, V. 2003. Management of Tetranychus urticae in Citrus in Spain acarofauna associated to weed. Bull.26: 213-220.

Awad, S.E., Mostafa, E.M., Salem, A.A., and Mahrous, M.E. 2018. Development and reproduction of the two-spotted red spider mite, Tetranychus urticae koch as influenced by feeding on leaves of three solanaceous vegetable crops under laboratory condition. Journal of Entomology. 15: 69-74

Blank, A.F., Silva, P.A., Arrigoni-Blank, M.F., Silva-Mann, R., and Barreto, M.C.V. 2005. Influência da adubaçãoorgânica e mineral no cultivo de manjericão cv. Genovese. Revista Ciencia Agronomica Journal. 36:175-180.

Boubou, A., Migeon, A., Roderick, G.K., and Navajas, M. 2011. Recent emergence and worldwide spread of the red tomato spider mite, Tetranychus evansi: genetic variation and multiple cryptic invasions. Biological Invasion. 13:81-92.

Boyd, D.W., and Alverson, D.R. 2000. Repellency effects of garlic extracts on two 
spotted spider mite, Tetranychus urticae Koch. Journal of Entomological Science. 35: 86-90.

Bussaman, C.S., Paweena, R., and Angsumarn, C. 2012. Effect of Crude Plant Extracts on Mushroom Mite, Luciaphorus sp. (Acari: Pygmephoridae) Prapassorn. Psyche: Journal of Entomology Pp. 1-5.

Chayengia, B., Patgiri, P., Rahman, Z., and Sarma, S. 2010. Efficacy of different plant products against Sitophilus oryzae (Linn.) (Coleoptera: Curculionidae) infestation on stored rice. Journal of Biopesticides. 3(3): 604-609.

Chiasson, H., Be'langer, A., Bostanian, N., Vincent, C., and Poliquin, A. 2001. Acaricidal properties of Artemisia absinthium and Tanacetum vulgare (Asteraceae) essential oils obtained by different methods of extraction. Journal of Economic Entomology. 94: 167-171

Deshpande, R.S., and Tipnis, H.P. 1997. Insecticidal activity of Ocimum basilieum L. Pesticides. 11: 11-12.

Geroh, M. 2011. Molecular Characterization of Beauveria bassiana (Balsamo) Vuillemin and its Bioefficacy against Tetranychus urticae Koch (Acari: Tetranychidae), Ph. D. Thesis, CCS HAU, Hisar.

Gogoi, D.V., Singh, and Srivastava, K.D. 2001. Phenols as a biochemical basis of resistance in wheat against Karnal bunt. Plant Pathology. 50: 470-476

Isabel, M.G., Antonio, Euzébio, M.G., Felipe, S.T.M., Wbyratan, L.V., and Liziane, M.S. et al., 2016. Bioactivity of basil (Ocimum basicilum L.) on control of the spider mite (Tetranychus urticae Koch.) in peanut. African Journal of Biotechnology. 15(30): 1597-1607.

Islam, M.T., Haque, M.M., Naher, N., and Parween, S. 2008. Effect of plant materials on the developmental stages of two spotted Spider mite Tetranychus urticae Koch (Acari: Tetranychidae). Journal of Biological Sciences. 16: 12-124

Kamaraj, C., Rahuman, A.A., and Bagavan, A. 2008. Antifeedant and larvicidal effects of plant extracts against Spodoptera litura (F.), Aedes aegypti L. and Culex quinquefasciatus Say. Parasitology
Research. 103: 325-331.

Kanika, Gulati, R., and Geroh, M. 2013. Influence of abiotic stresses on population dynamics of two spotted spider mite (Tetranychus urticae Koch) in Cucumber ecosystem. Annals of Plant Protection Sciences. 2 (2): 242-246.

Kanniammal, R., and Chinniah, C. 2012. Studies on exploitation of indigenous medicinal herbs for the management of two spotted spider mite, Tetranychus urticae Koch on okra and broad mite of chilli Polyphagotarsonemus latis Banks Msc. (Agri.) Thesis, submitted to Tamil Nadu Agricultural University, Maduri.

Kim, S.I., Roh, J.Y., Kim, D.H., Lee, H.S., and Ahn, Y.J. 2003. Insecticidal activity of aromatic plant extracts and essential oils against Sitophilus oryzae and Callosobruchus chinensis. Journal of Stored Product Research. 39: 293-303.

Kumar, R., Singh, R., Bharti, S.P., Kalidhar, S.B. 2001. Studies on insecticidal properties of Melia azedarach Seeds on Earias vittella. Journal of Cotton Research and Development. 15(2): 156-161.

Lange, W.H., and Bronson, L. 1981. Insect pests of tomatoes. Annual Review of Entomology. 26: 345-371.

Magdas, C., Cernea, M., Baciu, H., and Suteu, E. 2010. Acaricidal effect of eleven essential oil against the poultry mite Dermanyssus gallinae (Acari: Dermanyssidae). Scientica Parasitologica. 11(2): 71-75.

Manzoor, F., Beena, W., Malik, S., Naz N., Naz S, et al., 2011. Preliminary evaluation of Ocimum sanctum as toxicant and repellent against termite, Heterotermes indicola (Wasmann) (Isoptera: Rhinotermitidae). Pakistan Journal of Science. 63: 59-62.

Martinez-Villar, E., Saenz-de-Cabezon, F., Moreno-Grijalba, F., Marco, V., and PerezMoreno, I. 2005. Effects of azadirachtin on the two-spotted spider mite, Tetranychus urticae (Acari: Tetranychidae). Experimental and Applied Acarology. 35: 215-222.

Mehta, P.K. 2012. Scenario of insect pest problems under protected environment. In: Mehta et al., (Eds.) Pest management in high value crops under protected 
environment ICAR Short Course Department of Entomology, CSK Himachal Pradesh Krishi Vishvavidyalaya, Palampur, India. Pp.181.

Mishra, P, Mishra, S. 2011. Study of antibacterial activity of Ocimum sanctum extract against Gram positive and Gram negative bacteria. American Journal of Food Technology. 6(4): 336-341.

Mitra, S., Gupta, S.K., Ghosh, S. 2015. Bioefficacy of some green pesticides towards mortality and repellency against Petrobia harti Ewing (Acari: Tetranychidae) infesting medicinal weed, Oxalis corniculata L. (Oxalidaceae). International Journal of Applied Research. 1(11): 739742.

Nanasombat, S., and Lohasupthawee, P. 2005. Antibacterial activity of crude ethanolic extracts and essential oils of spices against Salmonellae and other Enterobacteria. KMITL Science and Technology Journal. 5(3): 527- 538.

Ogayo, K., Nyaanga, J., Ogweno, J., and Ogendo, J. 2019. The effect of lion's ear (Leonotis nepetifolia) and African Basil (Ocimum gratissimum) plant extracts on two-spotted spider mites (Tetranychus urticae) for Improved Yield and Quality of French Beans. Advances in Entomology. 7: 21-31.

Ogendo, J.O., Kostyukovsky, M., Ravid, U., Matasyoh, J.C., Deng, A.L., Omolo, E.O., Kariuki, S.T., and Shaaya, E. 2008. Bioactivity of Ocimum gratissimum L. oil and two of its constitutes against five insect pests attacking stored products. Journal of
Stored Products Research. 44: 328-334.

Roy, I., Aditya, G., and Saha, G.K. 2011. Preliminary assessment of selected botanicals in the control of Tetranychus neocaledonicus Andre (Acari: Tetranychidae), Proceedings of the Zoological Society. 64(2):124- 127.

Rusch, A., Valantin-Morison, M., Sarthou, J., and Roger-Estrade, J. 2010. Biological Control of Insect Pests in Agroecosystems: Effects of Crop Management, Farming Systems, and Semi-natural Habitats at the Landscape Scale: A Review. Advances in Agronomy. 109: 219-248.

Sahayaraj, K., Prabhu, J.N., and Martin, P. 2003. Screening of mite resistant okra varieties based on morphology and phytochemistry. Shashpa. 10(2): 147-150.

Sonika, Gulati, R.,and Jangra, M. 2017. Incidence of Tetranychus urticae Koch on brinjal under field and screen house conditions. Emergent Life Sciences Research. 3(2): 1622.

Spochacz, M., Chowanski, S., Walkowiak, N.K., Szymczak, M., and Adamski, Z. 2018. Plant- derived substances used against beetles- pests of stored crops and food and their mode of action: A review. Comprehensive reviews in Food science and Food safety.17: 1339-1366.

Uddin, M.N., Alam M.Z., Miah, M.R.U., Mian, M.I.H., Mustarin, K.E. 2015. Life Table Parameters of Tetranychus urticae Koch (Acari: Tetranychidae) on Different Bean Varieties. African Entomology. 23(2):418426.

\section{How to cite this article:}

Sonika Khatak, Rachna Gulati, Monika Jangra and Anju. 2020. In vitro Comparative Efficacy of Various Concentrations of Aqueous and Methanolic Leaf Extract of Ocimum sanctum against Tetranychus urticae Koch in Tomato. Int.J.Curr.Microbiol.App.Sci. 9(05): 2266-2275. doi: https://doi.org/10.20546/ijcmas.2020.905.258 